\title{
Supercyclicity and Resolvent Condition for Weighted Composition Operators
}

\author{
Tesfa Mengestie $^{1} \cdot$ Werkaferahu Seyoum $^{2}$
}

Received: 6 May 2020 / Revised: 6 December 2020 / Accepted: 21 December 2020 /

Published online: 8 April 2021

(C) The Author(s) 2021

\begin{abstract}
For pairs of holomorphic maps $(u, \psi)$ on the complex plane, we study some dynamical properties of the weighted composition operator $W_{(u, \psi)}$ on the Fock spaces. We prove that no weighted composition operator on the Fock spaces is supercyclic. Conditions under which the operators satisfy the Ritt's resolvent growth condition are also identified. In particular, we show that a non-trivial composition operator on the Fock spaces satisfies such a growth condition if and only if it is compact.
\end{abstract}

Keywords Fock spaces · Weighted composition operators · Supercyclic · Hypercyclic · Ritt resolvent condition - The unconditional Ritt's condition

Mathematics Subject Classification Primary 47B32 - 30H20; Secondary 46E22 . 46E20 - 47B33

\section{Introduction}

For pairs of holomorphic maps $(u, \psi)$, several properties of the weighted composition operator $W_{(u, \psi)}: f \rightarrow u \cdot f \circ \psi$ in various settings are well understood. But there are still some basic structures of the operators that require further investigation. In this paper, we study some dynamical structures of the operators on the Fock spaces

Communicated by Pekka Koskela.

$\bowtie \quad$ Tesfa Mengestie

Tesfa.Mengestie@hvl.no

Werkaferahu Seyoum

Werkaferahu@gmail.com

1 Department of Mathematical Sciences, Western Norway University of Applied Sciences,

Klingenbergvegen 8, 5414 Stord, Norway

2 Department of Mathematics, Addis Ababa University, Addis Ababa, Ethiopia 
$\mathcal{F}_{p}, 0<p<\infty$. We recall that the space $\mathcal{F}_{p}$ is the space consisting of all entire functions $f$ for which

$$
\|f\|_{p}^{p}=\frac{p}{2 \pi} \int_{\mathbb{C}}|f(z)|^{p} e^{-p|z|^{2} / 2} d A(z)<\infty
$$

where $d A$ is the usual Lebesgue area measure on the complex plane $\mathbb{C}$.

The space $\mathcal{F}_{2}$ is a reproducing kernel Hilbert space with kernel function $K_{w}(z)=$ $e^{\bar{w} z}$ and normalized kernel $k_{w}=\left\|K_{w}\right\|_{2}^{-1} K_{w}$. A straightforward calculation shows that for each $w \in \mathbb{C}$, the function $k_{w}$ belongs to $\mathcal{F}_{p}$ with $\left\|k_{w}\right\|_{p}=1$ for all $p$.

For each entire function $f$, the subharmonicity of $|f|^{p}$ implies the point estimate

$$
|f(z)| \leq e^{|z|^{2} / 2}\left(\int_{D(z, 1)}|f(w)|^{p} e^{-p|w|^{2} / 2} d A(w)\right)^{1 / 2} \leq e^{|z|^{2} / 2}\|f\|_{p}
$$

where $D(z, 1)$ is a disc of radius 1 and center $z$.

The bounded and compact weighted composition operators on Fock spaces were characterized in terms of Berezin-type integral transforms in $[9,20]$ and the reference therein. Later, Le [5] considered the Hilbert space $\mathcal{F}_{2}$ and obtained a simpler condition namely that $W_{(u, \psi)}$ is bounded on $\mathcal{F}_{2}$ if and only if $u \in \mathcal{F}_{2}$ and

$$
M(u, \psi):=\sup _{z \in \mathbb{C}}|u(z)| e^{\left(|\psi(z)|^{2}-|z|^{2}\right) / 2}<\infty .
$$

He further proved that (1.2) implies $\psi(z)=a z+b$ for some complex numbers $a$ and $b$ such that $|a| \leq 1$. In [14], Mengestie and Worku proved that the Berezintype integral condition used to describe the boundedness of generalized Volterra-type integral operators $V_{(g, \psi)}$ on the Fock spaces $\mathcal{F}_{p}$ is equivalent to a simple condition similar to (1.2). Because of the Littlewood-Paley type description of the Fock spaces, by simply replacing $\left|g^{\prime}(z)\right| /(1+|z|)$ by $|u(z)|$ in the results there, it has been known that (1.2) in fact describes the bounded weighted composition operators on all the spaces $\mathcal{F}_{p}, 1 \leq p<\infty$, with norm bounds

$$
M(u, \psi) \leq\left\|W_{(u, \psi)}\right\| \leq|a|^{-2 / p} M(u, \psi) .
$$

As indicated in [5], an interesting consequence of (1.2) is that if $|a|=1$, then a simple argument with Liouville's theorem leads to the explicit expression for the weight function

$$
u(z)=u(0) K_{-\bar{a} b}(z) .
$$

We note that $W_{(u, \psi)}$ can be written as the product $M_{u} C_{\psi}$ where $M_{u}$ and $C_{\psi}$ are respectively the multiplication and composition operators. By condition (1.2), the operator $W_{(u, \psi)}$ can be bounded even if both the factors $C_{\psi}$ and $M_{u}$ are unbounded. For example one can set $u_{0}(z)=e^{-z}, \psi_{0}(z)=z+1$, and observe that $W_{\left(u_{0}, \psi_{0}\right)}$ is bounded while both the factors remain unbounded. Similarly, compactness of $W_{(u, \psi)}$ has been 
described by the fact that $\psi(z)=a z+b,|a| \leq 1$ and $|u(z)| e^{\left(|\psi(z)|^{2}-|z|^{2}\right) / 2} \rightarrow 0$ as $|z| \rightarrow 0$. Compactness implies that $|a|<1$.

We conclude this section with a word on notation. The notation $U(z) \lesssim V(z)$ (or equivalently $V(z) \gtrsim U(z))$ means that there is a constant $C$ such that $U(z) \leq C V(z)$ holds for all $z$ in the set of a question. We write $U(z) \simeq V(z)$ if both $U(z) \lesssim V(z)$ and $V(z) \lesssim U(z)$.

\section{Supercyclic Weighted Composition Operators on $\mathcal{F}_{p}$}

The power bounded and uniformly mean ergodic dynamical properties of the weighted composition operators on $\mathcal{F}_{p}$ have been recently described in [18]. One of the main objectives of this note is to take that investigation further and study the supercyclic structure and resolvent growth conditions of the operators on the spaces. We start by recalling certain definitions. A bounded linear operator $T$ on a Banach space $\mathcal{X}$ is said to be hypercyclic if there exists a vector $f$ in $\mathcal{X}$ for which the orbit, $\operatorname{Orb}(T, f)=$ $\left\{T^{n} f: n \in \mathbb{N}_{0}\right\}$, is dense in $\mathcal{X}$ where the operator $T^{n}$ is the $n$-th iterate of $T, T^{0}=I$ and $I$ is the identity map on $\mathcal{X}$. The operator is supercyclic if there exists a vector $f$ for which its projective orbit, the set of scalar multiplies of $\operatorname{Orb}(T, f)$, is dense in $\mathcal{X}$. Clearly, every hypercyclic operator is supercyclic but not conversely.

We note in passing that the dynamical properties of operators have been the object of many investigations over the past several years in part because these properties are intimately connected with the study of the famous invariant subspace open problem .

The study of the dynamical properties of an operator is related to the study of its iterates. For the operator $W_{(u, \psi)}$ and an entire function $f$, a simple computation shows that each element of the orbit has the form

$$
W_{(u, \psi)}^{n} f=f\left(\psi^{n}\right) u_{n}, \quad u_{n}:=\prod_{j=0}^{n-1} u\left(\psi^{j}\right)
$$

for each $n \in \mathbb{N}$ and $\psi^{0}=I$ the identity map on $\mathbb{C}$. In [12,13], it was shown that the Fock spaces support no supercyclic and hence hypercyclic composition operators. Motivated by this, one may ask whether the interplay between $u$ and $\psi$ in (2.1) results in supercyclic weighted composition operators on Fock spaces as it does for example in the case of boundedness and compactness operator theoretic structures. Our next main result affirms that the spaces support no supercyclic weighted composition operator either.

Theorem 2.1 Let $1 \leq p<\infty$ and $(u, \psi)$ be a pair of entire functions on $\mathbb{C}$ which induces a bounded weighted composition operator $W_{(u, \psi)}$ on $\mathcal{F}_{p}$. Then $W_{(u, \psi)}$ can not be supercyclic on $\mathcal{F}_{p}$.

As pointed above if $u=1$, then $W_{(u, \psi)}$ is just the composition operator $C_{\psi}$. On the other hand, if $\psi$ is the identity map, then $W_{(u, \psi)}$ reduces to the multiplication operator $M_{u}$. With this, we obtain the following immediate consequence of Theorem 2.1. 
Corollary 2.2 Let $1 \leq p<\infty$ and $(u, \psi)$ be a pair of entire functions on $\mathbb{C}$, and let the operators $C_{\psi}$ and $M_{u}$ be bounded on $\mathcal{F}_{p}$. Then, both $C_{\psi}$ and $M_{u}$ are not supercyclic on $\mathcal{F}_{p}$.

The results above assert that the projective orbit of any given vector under $W_{(u, \psi)}$ is not large enough to contain the space $\mathcal{F}_{p}$. The weighted operators exhibit the same supercyclic phenomena as the unweighted composition operator $C_{\psi}$. Since the weight function had no effect on this property here, it is interesting to ask what type of general Fock spaces do support supercyclic composition operators. When the weight functions generating the spaces grow faster than the classical weight function $|z|^{2} / 2$, it was verified in [14] that the corresponding Fock-type spaces fail to support. If the weight functions grow slower than the classical case and the derivative of the weight function grows to infinity, the approaches used in [14] show that the same conclusion follows. On the other hand, when the weight function is $\psi_{m}(z)=|z|^{m}, 0<m \leq 1$, then as shown in [4], each non-trivial translation operator acting on the corresponding Fock-type spaces is hypercyclic and hence is supercyclic. Since a bounded $C_{\psi}$ on such spaces happens if and only if $\psi(z)=a z+b$ with $|a| \leq 1$, by setting $a=1$ and $b \neq 0$, we observe that $C_{\psi}$ reduces to the translation operator $T_{b}$ which is hypercyclic. This in fact gives spaces where the composition operator admits hypercyclic structure and hence supercyclic. Whether this extends to the non-trivial weighted composition operators remains to be studied.

Proof of Theorem 2.1. We now give the proof of the theorem. Since $W_{(u, \psi)}$ is bounded, we set $\psi(z)=a z+b$, with $|a| \leq 1$. For the case $|a|<1$ or $|a|=1$ and $a \neq 1$, we argue as follows. The map $\psi$ fixes the point $z_{0}=b /(1-a)$. Assume on the contrary that there exists a supercyclic vector $f$ in $\mathcal{F}_{p}$. First we claim that $u$ is zero free on $\mathbb{C}$ because if $\mathrm{u}$ vanishes at the point $w$, then (2.1) implies that every element in the orbit of $f$ vanishes at $w$ which extends to the projective orbit which obviously is not the case. Observe also that $f$ can not have a zero in $\mathbb{C}$. This is because all the elements in the projective orbit will also vanish at a possible zero which extends to the closure and gives a contradiction. Thus, by Proposition 4 of [2], for any two different numbers $z, w \in \mathbb{C}$,

$$
\overline{\left\{\frac{u_{n}(z) f\left(\psi^{n}(z)\right)}{u_{n}(w) f\left(\psi^{n}(w)\right)}\right\}}=\mathbb{C} .
$$

Let $r>0$ be given. Then $K=\left\{z \in \mathbb{C}:\left|z-z_{0}\right| \leq r\right\}$ is a compact neighbourhood of $z_{0}$ which also contains $\psi(K)$ since for each $z \in K$

$$
\left|\psi(z)-z_{0}\right|=\left|a z+b-z_{0}\right| \leq\left|a z-a z_{0}\right|+\left|a z_{0}-z_{0}+b\right| \leq|a| r \leq r .
$$

Now, if we set $w=\psi(z), z \in K, z \neq z_{0}$ and consider the expression in (2.2)

$$
\left|\frac{u_{n}(z) f\left(\psi^{n}(z)\right)}{u_{n}(w) f\left(\psi^{n}(w)\right)}\right|=\left|\frac{u(z) f\left(\psi^{n}(z)\right)}{u\left(\psi^{n}(z)\right) f\left(\psi^{n+1}(z)\right)}\right| \leq C
$$


for all $n \in \mathbb{N}$ where

$$
C=\frac{\max _{z \in K}|u(z)| \cdot \max _{z \in K}|f(z)|}{\min _{z \in K}|u(z)| \cdot \min _{z \in K}|f(z)|}
$$

This obviously contradicts the relation in (2.2).

It remains to prove the case for $a=1$. This is rather immediate as by (1.4), we have $u(z)=u(0) K_{-b}(z)$ and

$$
W_{(u, \psi)} f=u(0) K_{-b} f(\psi)=u(0)\left\|K_{-b}\right\|_{2} k_{-b} f(\psi)=u(0) e^{|b|^{2} / 2} W_{\left(k_{-b}, \psi\right)} f .
$$

Then we show that $W_{\left(k_{-b}, \psi\right)}$ is an isometry. Thus, for $f \in \mathcal{F}_{p}$

$$
\begin{aligned}
\left\|W_{\left(k_{-b}, \psi\right)} f\right\|_{p}^{p}= & \frac{p}{2 \pi}\left\|K_{-b}\right\|_{2}^{-p} \int_{\mathbb{C}}\left|K_{-b}(z)\right|^{p}|f(z+b)|^{p} e^{-p / 2|z|^{2}} d A(z) \\
= & \frac{p}{2 \pi}\left\|K_{-b}\right\|_{2}^{-p} \int_{\mathbb{C}}|f(z+b)|^{p} e^{-p|z+b|^{2} / 2} \\
& \times\left(\left|K_{-b}(z)\right|^{p} e^{p|z+b|^{2} / 2-p|z|^{2} / 2}\right) d A(z) \\
= & \left\|K_{-b}\right\|_{2}^{-p} e^{p|b|^{2} / 2}\|f\|_{p}^{p}=\|f\|_{p}^{p}
\end{aligned}
$$

for all $1 \leq p<\infty$. This shows that the operator is a linear isometry, and in fact one can easily show that it is also a bijective operator with inverse map $W_{\left(k_{-b}, \psi\right)}^{-1}=W_{\left(k_{b}, \psi^{-1}\right)}$.

It follows from this, the fact that $u(0) \neq 0$ and $(2.3)$, that $W_{(u, \psi)}$ is a normal operator. Then the conclusion of supecyclicity follows directly from the well known Ansari-Bourdon's theorem [1] and completes the proof.

\section{The Ritt's Resolvent Growth Condition for $W_{(u, \psi)}$ on $\mathcal{F}_{p}$}

The resolvent growth condition for an operator is another dynamical structure closely related to its iterates, power boundedness and spectral properties. We recall that the resolvent function $R(\lambda, T)$ of an operator $T$ defined on the resolvent set $\varrho(T)$ is an operator valued function given by $R(\lambda, T)=(\lambda I-T)^{-1}$. If $T$ is power bounded and hence $\sigma(T)$ contained in the closed unit disc, then using the series expansion of the resolvent function

$$
\|R(\lambda, T)\|=\left\|\sum_{n=0}^{\infty} \frac{T^{n}}{\lambda^{n+1}}\right\| \leq \sum_{n=0}^{\infty} \frac{\left\|T^{n}\right\|}{|\lambda|^{n+1}}=\frac{c}{|\lambda|-1}
$$

where $c:=\sup _{n \in \mathbb{N}_{0}}\left\|T^{n}\right\|$. Thus, every power bounded operator satisfies the so-called Kreiss's resolvent condition. Conversely, for infinite dimensional spaces, condition (3.1) does not imply power boundedness as it only gives $\left\|T^{n}\right\|=O(n)$ as $n \rightarrow \infty$; see for example [19]. Now we are interested in a stronger resolvent growth condition that conversely implies power boundedness, namely, the Ritt's resolvent condition 
[17]. An operator $T$ satisfies such a condition if there exists a positive constant $M$ such that

$$
\|R(\lambda, T)\| \leq \frac{M}{|\lambda-1|}
$$

for all $\lambda \in \mathbb{C}$ with $|\lambda|>1$. Both the Kreiss's and Ritt's resolvent conditions play important roles in numerical analysis; see [3] and the references therein for more details.

We now state our next main result.

Theorem 3.1 Let $1 \leq p<\infty$ and $(u, \psi)$ be a pair of entire functions on $\mathbb{C}$ which induces a bounded weighted composition operator $W_{(u, \psi)}$ on $\mathcal{F}_{p}$ and hence $\psi(z)=$ $a z+b,|a| \leq 1$. Then if

(i) $|a|=1$, then $W_{(u, \psi)}$ satisfies the Ritt's resolvent condition if and only if any one of the following holds:

- $a=1, b=0$ and $|u(0)|<1$

- $a=1, b=0$ and $u(0)=1$

- $a=1, b \neq 0$ and $|u(0)|<e^{-|b|^{2} / 2}$

- $a \neq 1$ and $|u(0)|<e^{-|b|^{2} / 2}$.

(ii) $W_{(u, \psi)}$ is compact and satisfies the Ritt's resolvent growth condition, then $|u(b /(1-a))| \leq 1$. Conversely, if $|u(b /(1-a))|<1$, then $W_{(u, \psi)}$ satisfies the Ritt's resolvent growth condition.

Proof In 1999, Nagy and Zemanek [15] proved that a bounded operator $T$ on complex Banach space satisfies the Ritt's resolvent growth condition if and only if it is power bounded and the difference of its consecutive iterates satisfy

$$
\sup _{n \in \mathbb{N}} n\left\|T^{n+1}-T^{n}\right\|<\infty .
$$

As before, we let $\psi(z)=a z+b,|a| \leq 1$, and set $z_{0}=b /(1-a)$ when $a \neq 1$. From [18, Thm. 3.1], we recall the following results about the spectrum of the operators. If $W_{(u, \psi)}$ is compact and hence $|a|<1$, then

$$
\sigma\left(W_{(u, \psi)}\right)=\left\{0, u\left(z_{0}\right) a^{m}, \quad m \in \mathbb{N}_{0}\right\} .
$$

On the other hand, if $|a|=1$, then

$$
\sigma\left(W_{(u, \psi)}\right)=\left\{\begin{array}{l}
\overline{\left\{u\left(z_{0}\right) a^{m}: m \in \mathbb{N}_{0}\right\}}, a \neq 1 \\
\left\{z \in \mathbb{C}:|z|=|u(0)| e^{|b|^{2} / 2}\right\}, \quad a=1, \quad b \neq 0 \\
\{u(0)\}, \quad a=1, \quad b=0 .
\end{array}\right.
$$

Now from [6] and [16, Thm. 4.5.4], if $W_{(u, \psi)}$ satisfies the Ritt's growth condition, then its spectrum is contained in a stolz type domain $\overline{\beta_{\theta}}$ where $\beta_{\theta}$ is the interior of convex 
hull of the set $\{1\}$ and the disc $\{z \in \mathbb{D}:|z| \leq \sin \theta\}$ and $\theta=\arccos (1 / M)$ where $M$ is the best possible constant in (3.2) and $\theta \in[0, \pi / 2)$. In particular, we have

$$
\sigma\left(W_{(u, \psi)}\right) \cap \mathbb{T} \subset\{1\}
$$

where $\mathbb{T}$ denotes the unit circle.

(i) Since Ritt's condition implies power boundedness, by [18, Thm. 2.1],

$$
\left|u\left(\frac{b}{1-a}\right)\right|=|u(0)| e^{|b|^{2} / 2} \leq 1 .
$$

This together with (3.5) and (3.6) implies for $|a|=1$ that either $a=1, b=0$ and $|u(0)| \leq 1$ or $u(0)=1$, or $a=1, b \neq 0$ and $|u(0)|<e^{-|b|^{2} / 2}$ or $a \neq 1$ and $|u(0)|<e^{-|b|^{2} / 2}$.

Conversely, if $a=1, b=0$ and $|u(0)| \leq 1$, then $W_{(u, \psi)}$ is trivially power bounded. Furthermore, $W_{(u, \psi)}$ reduces to the multiplication operator $M_{u}$. By [11, Lem. 2.3], it is known that $M_{u}$ is bounded on $\mathcal{F}_{p}$ if and only if $u$ is a constant function. Thus, if $u=u(0)$, then $\left\|T^{n+1}-T^{n}\right\|=|u(0)|^{n}|1-u(0)|$ which implies that condition (3.3) holds whenever $u(0)=1$ or $|u(0)|<1$. On the other hand, if $|u(0)| \leq 1$ or $a=1$, $b \neq 0$ and $|u(0)|<e^{-|b|^{2} / 2}$ or $a \neq 1$ and $|u(0)|<e^{-|b|^{2} / 2}$, then applying (1.3) and (1.4), we have

$$
\begin{aligned}
\left\|W_{(u, \psi)}^{n}\right\|=\left|u\left(z_{0}\right)\right|^{n} & =\left|u(0) e^{a|b|^{2} /(a-1)}\right|^{n}=\left(|u(0)| e^{|b|^{2} \operatorname{Re}(a /(a-1))}\right)^{n} \\
& =|u(0)|^{n} e^{n|b|^{2} \operatorname{Re}(a(\bar{a}-1) /[(a-1)(\bar{a}-1)])}=|u(0)|^{n} e^{n|b|^{2} / 2},
\end{aligned}
$$

from which it follows that

$$
\sup _{n \in \mathbb{N}} n\left\|W_{(u, \psi)}^{n+1}-W_{(u, \psi)}^{n}\right\| \leq \sup _{n \in \mathbb{N}} n\left(|u(0)| e^{|b|^{2} / 2}\right)^{n}+\sup _{n \in \mathbb{N}} n\left(|u(0)| e^{|b|^{2} / 2}\right)^{n+1}<\infty .
$$

(ii) For $|a|<1$, first observe that for each $n \in \mathbb{N}$, the operator $W_{(u, \psi)}^{n}$ itself is a weighted composition operator induced by the symbol $\left(u_{n}, \psi^{n}\right)$ and $W_{(u, \psi)}^{n}=W_{\left(u_{n}, \psi^{n}\right)}$. Aiming to check condition (3.3), we first find an estimate for the norm of the difference of the two weighted composition operators,

$$
\left\|W_{(u, \psi)}^{n+1}-W_{(u, \psi)}^{n}\right\|=\left\|W_{\left(u_{n+1}, \psi^{n+1}\right)}-W_{\left(u_{n}, \psi^{n}\right)}\right\| .
$$

Applying the difference to the normalized reproducing kernels $k_{w}$ and relation (1.1)

$$
\begin{aligned}
\left\|W_{\left(u_{n+1}, \psi^{n+1}\right)}-W_{\left(u_{n}, \psi^{n}\right)}\right\| & \geq\left\|W_{\left(u_{n+1}, \psi^{n+1}\right)} k_{w}-W_{\left(u_{n}, \psi^{n}\right)} k_{w}\right\|_{p} \\
& \geq\left|u_{n+1}(z) e^{\bar{w} \psi^{n+1}(z)}-u_{n}(z) e^{\bar{w} \psi^{n}(z)}\right| e^{-\left(|z|^{2}+|w|^{2}\right) / 2}
\end{aligned}
$$


for all $w, z \in \mathbb{C}$. In particular, setting $z=w=z_{0}$ it readily follows from (3.7) and (2.1) that

$$
\left\|W_{\left(u_{n+1}, \psi^{n+1}\right)}-W_{\left(u_{n}, \psi^{n}\right)}\right\| \geq\left|u_{n}\left(z_{0}\right)\right|\left|u\left(z_{0}\right)-1\right|=\left|u\left(z_{0}\right)\right|^{n}\left|u\left(z_{0}\right)-1\right|,
$$

from which the relation in (3.3) for $\left\|W_{(u, \psi)}\right\|$ holds only if $\left|u\left(z_{0}\right)\right| \leq 1$.

Conversely, by [18, Thm. 2.2], $W_{(u, \psi)}$ is power bounded if and only if $\left|u\left(z_{0}\right)\right| \leq 1$, and $\left\|W_{(u, \psi)}^{n}\right\| \simeq\left|u\left(z_{0}\right)\right|^{n}$. Then we repeat the arguments made for $|a|=1, a \neq 1$ to show that (3.3) holds whenever $\left|u\left(z_{0}\right)\right|<1$, and this completes the proof.

It would be desirable to know whether the necessity condition $\left|u\left(z_{0}\right)\right|=1$ could be sufficient as well. We in fact conjecture that it should be. In the following we provide several results in favor of our conjecture.

Corollary 3.2 Let $1 \leq p<\infty$ and $(u, \psi)$ be a pair of entire functions on $\mathbb{C}$ which induces a bounded weighted composition operator $W_{(u, \psi)}$ on $\mathcal{F}_{p}$ and hence $\psi(z)=$ $a z+b,|a| \leq 1$. If $a=0$, then $W_{(u, \psi)}$ satisfies the Ritt's resolvent condition on $\mathcal{F}_{p}$ if and only if either $u(b)=1$ or $|u(b)|<1$.

Proof. The necessity of the condition follows from (3.8) as a particular case. Thus, we shall verify the sufficiency. A simple computation shows that for each $f \in \mathcal{F}_{p}$

$$
\left\|W_{\left(u_{n+1}, \psi^{n+1}\right)} f-W_{\left(u_{n}, \psi^{n}\right)} f\right\|_{p}^{p} \leq|u(b)|^{p n}\|f\|_{p}^{p}|u(b)-1|^{p} .
$$

It follows that

$$
\left\|W_{\left(u_{n+1}, \psi^{n+1}\right)}-W_{\left(u_{n}, \psi^{n}\right)}\right\| \leq|u(b)|^{n}|u(b)-1|
$$

from which it is easy to see that (3.3) holds.

Observe that the multiplication operator $M_{u}$ is power bounded if and only if $|u(0)| \leq$ 1. This gives the following consequence.

Corollary 3.3 Let $1 \leq p<\infty$ and $u$ be an entire functions on $\mathbb{C}$. Then a non-trivial $M_{u}$ satisfies the Ritt's resolvent condition on $\mathcal{F}_{p}$ if and only if it is power bounded.

The composition operator is one of the other cases where we have $u\left(z_{0}\right)=1$. For this we show that the Ritt's resolvent condition is in fact equivalent to the stronger unconditional Ritt's condition on $\mathcal{F}_{p}$. We recall that an operator $T$ on a Banach space $\mathcal{X}$ satisfies the unconditional Ritt's condition if there exists a non-negative constant $\mathrm{K}$ such that

$$
\left\|\sum_{n=1} a_{n}\left(T^{n}-T^{n-1}\right)\right\| \leq K \sup _{n}\left\{\left|a_{n}\right|\right\}
$$

for any finite sequence $\left(a_{n}\right)$ of complex numbers. We note in passing that the notion of the unconditional Ritt's condition is the discrete analogue of the $H^{\infty}$ calculus for sectorial operators [8]. Kalton and Portal [7] proved that the unconditional Ritt's condition implies the Ritt's resolvent condition in general, but not conversely. But for the composition operators, it turns out that the two are equivalent. 
Theorem 3.4 Let $1 \leq p<\infty$ and $\psi$ be an entire function on $\mathbb{C}$ which induces a bounded composition operator $C_{\psi}$ on $\mathcal{F}_{p}$. Then the following are equivalent.

(i) $C_{\psi}$ satisfies the Ritt's resolvent condition on $\mathcal{F}_{p}$;

(ii) $C_{\psi}$ is compact or $C_{\psi}$ is the identity map on $\mathcal{F}_{p}$;

(iii) $C_{\psi}$ satisfies the unconditional Ritt's condition on $\mathcal{F}_{p}$.

Proof For $\mathrm{p}=2$, the result was proved in [10]. We now modify the proof for all $p^{\prime} s$ and first show that the statements in (i) and (ii) are equivalent. Recall that $C_{\psi}$ is bounded if and only if $\psi(z)=a z+b,|a| \leq 1$ and $b=0$ whenever $|a|=1$. Compactness is described by the strict inequality $|a|<1$. If $|a|=1$, then by Theorem 3.1 the composition operator $C_{\psi}$ satisfies the Ritt's resolvent condition on $\mathcal{F}_{p}$ if and only if $a=1$, which means $\psi$ reduces to the identity operator. Thus, we shall proceed to show the equivalency for the case when $|a|<1$. The statement (i) implies (ii) follows again from part (ii) of Theorem 3.1. We need to prove the converse. For each $z \in \mathbb{C}$ and $n \in \mathbb{N}$, let $\delta_{n}$ be a positive number such that

$$
a^{n+1} z+\frac{b\left(1-a^{n+1}\right)}{1-a} \in D\left(a^{n} z+\frac{b\left(1-a^{n}\right)}{1-a}, \delta_{n}\right) .
$$

An explicit expression for $\delta_{n}$ will be given latter. Then for any $f \in \mathcal{F}_{p}$, by the Mean Value Theorem

$$
\begin{aligned}
\left|C_{\psi^{n+1}} f(z)-C_{\psi^{n}} f(z)\right|^{p}= & \left|f\left(a^{n+1} z+\frac{b\left(1-a^{n+1}\right)}{1-a}\right)-f\left(a^{n} z+\frac{b\left(1-a^{n}\right)}{1-a}\right)\right|^{p} \\
\leq & \left|a^{n+1} z+\frac{b\left(1-a^{n+1}\right)}{1-a}-a^{n} z-\frac{b\left(1-a^{n}\right)}{1-a}\right|^{p} \\
& \times \sup _{w \in D\left(a^{n} z+b\left(1-a^{n}\right) /(1-a), \beta_{n}\right)}\left|f^{\prime}(w)\right|^{p} \\
= & \left|a^{n} z(a-1)+b a^{n}\right|^{p} \sup _{w \in D\left(a^{n} z+b\left(1-a^{n}\right) /(1-a), \beta_{n}\right)}\left|f^{\prime}(w)\right|^{p}
\end{aligned}
$$

for some positive $\beta_{n}<\delta_{n}$ which will also be fixed latter.

Using the reproducing property,

$$
f(w)=\left\langle f, K_{w}\right\rangle=\frac{1}{\pi} \int_{\mathbb{C}} f(z) e^{w \bar{z}} e^{-|z|^{2}} d A(z)
$$

and

$$
\begin{aligned}
\left|f^{\prime}(w)\right| & \leq \frac{1}{\pi} \int_{\mathbb{C}}|z||f(z)| e^{\operatorname{Re}(w \bar{z})} e^{-|z|^{2}} d A(z) \\
& \leq \frac{1}{\pi}\left(\sup _{z \in \mathbb{C}}|f(z)| e^{-|z|^{2} / 2}\right) \int_{\mathbb{C}}|z| e^{\operatorname{Re}(w \bar{z})} e^{-|z|^{2} / 2} d A(z)
\end{aligned}
$$




$$
\begin{aligned}
& \leq \frac{1}{\pi}\|f\|_{p} \int_{\mathbb{C}}|z| e^{\operatorname{Re}(w \bar{z})} e^{-|z|^{2} / 2} d A(z) \\
& \leq \frac{1}{\pi} e^{|w|^{2} / 2}\|f\|_{p} \int_{\mathbb{C}}(1+|z|) e^{-|z-w|^{2} / 2} d A(z) .
\end{aligned}
$$

On the other hand,

$$
1+|z| \leq 1+|w-z|+|w| \leq(1+|w-z|)(1+|w|)
$$

and hence

$$
\begin{aligned}
\frac{1}{\pi} \int_{\mathbb{C}}(1+|z|) e^{-|z-w|^{2} / 2} d A(z) & \leq(1+|w|) \frac{1}{\pi} \int_{\mathbb{C}}(1+|w-z|) e^{-|z-w|^{2} / 2} d A(z \\
& =(1+|w|)\left(1+2^{\frac{3}{2}} \Gamma\left(1+\frac{1}{2}\right)\right) \\
& =(\sqrt{2 \pi}+1)(1+|w|)
\end{aligned}
$$

where $\Gamma$ refers to the gamma function. Thus, we deduce

$$
\left|f^{\prime}(w)\right| \leq(\sqrt{2 \pi}+1)(1+|w|) e^{|w|^{2} / 2}\|f\|_{p} .
$$

Now, since $w \in D\left(a^{n} z+b\left(1-a^{n}\right) /(1-a), \beta_{n}\right)$ we have

$$
\begin{aligned}
|w| & \leq\left|w-a^{n} z+\frac{b\left(1-a^{n}\right)}{1-a}\right|+\left|a^{n} z+\frac{b\left(1-a^{n}\right)}{1-a}\right| \\
& \leq \beta_{n}+\left|a^{n} z+\frac{b\left(1-a^{n}\right)}{1-a}\right| \leq \beta_{n}+|a|^{n}|z|+\frac{2|b|}{|1-a|}=: \alpha_{n}(z) .
\end{aligned}
$$

Now taking all these estimates in (3.10)

$$
\begin{aligned}
& \frac{1}{\pi} \int_{\mathbb{C}}\left|C_{\psi^{n+1}} f(z)-C_{\psi^{n}} f(z)\right|^{p} e^{-p / 2|z|^{2}} d A(z) \\
& \quad \leq 2|a|^{p n}\|f\|_{p}^{p}(\sqrt{2 \pi}+1)^{p} \int_{\mathbb{C}}|(a-1) z+b|^{p}\left|1+\alpha_{n}(z)\right|^{p} e^{p\left(\alpha_{n}^{2}(z)-|z|^{2}\right) / 2} d A(z) \\
& \quad \leq 2|a|^{p n}\|f\|_{p}^{p}(\sqrt{2 \pi}+1)^{p} I_{n}
\end{aligned}
$$

where

$$
I_{n}=\int_{\mathbb{C}}\left(|z|^{2}+|z|^{2} \alpha_{n}^{2}(z)+|b|^{2}+|b|^{2} \alpha_{n}^{2}(z)\right) e^{p\left(\alpha_{n}^{2}(z)-|z|^{2}\right) / 2} d A(z) .
$$

Next, we show that the integral above is uniformly bounded by a positive number independent of $n \in \mathbb{N}$. To this end, observe that since 


$$
\begin{aligned}
& a^{n+1} z+\frac{b\left(1-a^{n+1}\right)}{1-a} \in D\left(a^{n} z+\frac{b\left(1-a^{n}\right)}{1-a}, \delta_{n}\right) \\
& \left|a^{n+1} z+\frac{b\left(1-a^{n+1}\right)}{1-a}-a^{n} z-\frac{b\left(1-a^{n}\right)}{1-a}\right|=\left|a^{n} z(a-1)+b a^{n}\right| \\
& \quad \leq|a|^{n}|a-1||z|+|a|^{n}|b|
\end{aligned}
$$

$\delta_{n}$ can be taken to be $|a|^{n}|a-1||z|+|a|^{n}|b|$. Thus, $\beta_{n}$ can also be chosen in such a way that $\beta_{n}=\alpha_{n}|z|+|a|^{n}|b|$ for some $0<\alpha_{n}<|a|^{n}|a-1|$ whenever $z \neq 0$. Then

$$
\begin{aligned}
\alpha_{n}^{2}(z)< & \left(|a|^{n}(|a-1|+1)|z|+|a|^{n}|b|+\frac{2|b|}{|1-a|}\right)^{2}=|a|^{2 n}(|a-1|+1)^{2}|z|^{2} \\
& +2|a|^{n}|z|(|a-1|+1)\left(|a|^{n}|b|+\frac{2|b|}{|1-a|}\right)+\left(|a|^{n}|b|+\frac{2|b|}{|1-a|}\right)^{2}
\end{aligned}
$$

Given the estimate in (3.12) and the exponential integrating weight in (3.11), we claim that

$$
|a|^{2 n}(|a-1|+1)^{2}-1 \leq 0
$$

for all $n$. Setting $a=|a| e^{i \theta}$ we write

$$
|a|^{2 n}\left((|a-1|+1)^{2}-1=|a|^{2 n}\left(|a| \sqrt{(\cos \theta-1)^{2}+(\sin \theta)^{2}}+1\right)^{2}-1 .\right.
$$

Thus, it is enough to show that $|a|^{2}(2|a|+1)^{2}-1 \leq 0$. But this obviously holds since $r=|a|<1$ and the values of the function $g(r)=r^{2}(2 r+1)^{2}-1$ lie in the interval $[-1,0]$. Now, integrating with polar coordinates shows that the integral in (3.11) is uniformly bounded by a positive number $C$ independent of $n$. Therefore,

$$
\left\|C_{\psi^{n+1}}-C_{\psi^{n}}\right\|^{p} \leq C|a|^{p n}
$$

This together with $|a|<1$ implies that the relation in (3.3) holds for $C_{\psi}$.

Next we show that the statements in (i) and (iii) are also equivalent. From the discussion above, we have already mentioned that (iii) implies (i). Thus, it remains to show (i) implies (iii). But this is rather immediate since by (3.14), we observe that $\left\|C_{\psi^{n}}-C_{\psi^{n+1}}\right\|$ is bounded by an exponentially decreasing sequence with $n$. Thus

$$
\left\|\sum_{n=1} a_{n}\left(C_{\psi^{n}}-C_{\psi^{n-1}}\right)\right\| \leq \sup _{n}\left\{\left|a_{n}\right|\right\} \sum_{n=1}\left\|C_{\psi^{n}}-C_{\psi^{n-1}}\right\| \lesssim \sup _{n}\left\{\left|a_{n}\right|\right\} .
$$

Funding Open access funding provided by Western Norway University Of Applied Sciences. 
Open Access This article is licensed under a Creative Commons Attribution 4.0 International License, which permits use, sharing, adaptation, distribution and reproduction in any medium or format, as long as you give appropriate credit to the original author(s) and the source, provide a link to the Creative Commons licence, and indicate if changes were made. The images or other third party material in this article are included in the article's Creative Commons licence, unless indicated otherwise in a credit line to the material. If material is not included in the article's Creative Commons licence and your intended use is not permitted by statutory regulation or exceeds the permitted use, you will need to obtain permission directly from the copyright holder. To view a copy of this licence, visit http://creativecommons.org/licenses/by/4.0/.

\section{References}

1. Ansari, I., Bourdon, P.S.: Some properties of cyclic operators. Acta Sci. Math. (Szeged) 63(12), 195207 (1997)

2. Beltrán-Meneu, M.J., Jordá, E., Murillo-Arcila, M.: Supercyclicity of weighted composition operators on spaces of continuous functions. Collect. Math. 71, 493-509 (2020)

3. Borovykh, N., Drissi, D., Spijker, M.N.: A note about Ritt's condition, related resolvent conditions and power bounded operators. Numer. Funct. Anal. Optim. 21, 425-438 (2000)

4. Guo, K., Izuchi, K.: Composition operators on Fock type spaces. Acta Sci. Math. (Szeged) 74, 807-828 (2008)

5. Le, T.: Normal and isometric weighted composition operators on the Fock space. Bull. Lond. Math. Soc. 46, 847-856 (2014)

6. Lyubich, Yu.: Spectral localization, power boundedness and invariant subspaces under Ritt's type condition. Stud. Math. 143(2), 153-167 (1999)

7. Kalton, N.J., Portal, P.: Remarks on $\ell_{1}$ and $\ell_{\infty}$-maximal regularity for power bounded operators. J. Aust. Math. Soc. 84, 345-365 (2008)

8. McIntosh, A.: Operators which have an $H_{\infty}$ functional calculus. Miniconference on Operator Theory and Partial Differential Equations, pp. 210-231, Centre for Mathematics and its Applications, Mathematical Sciences Institute, The Australian National University, Canberra (1986)

9. Mengestie, T.: Carleson type measures for Fock-Sobolev spaces. Complex Anal. Oper. Theory 8(6), 1225-1256 (2014)

10. Mengestie, T.: Resolvent growth condition for composition operators on the Fock space. Ann. Funct. Anal. 11, 947-955 (2020)

11. Mengestie, T.: Spectral properties of Volterra-type integral operators on Fock-Sobolev spaces. J. Korean Math. Soc. 54(6), 1801-1816 (2017)

12. Mengestie, T., Seyoum, W.: Spectral properties of composition operators on Fock-type spaces. Quaest. Math. https://doi.org/10.2989/16073606.2019.1692092

13. Mengestie, T., Seyoum, W.: Topological and dynamical properties of composition operators. Complex Anal. Oper. Theory 14, 2 (2020). https://doi.org/10.1007/s11785-019-00961-8

14. Mengestie, T., Worku, M.: Topological structures of generalized Volterra-type integral operators. Mediterr. J. Math. 15, 42 (2018). https://doi.org/10.1007/s00009-018-1080-5

15. Nagy, B., Zemanek, J.A.: A resolvent condition implying power boundedness. Stud. Math. 134, 143151 (1999)

16. Nevanlinna, O.: Convergence of iterations for linear equations. Lecture Notes in Mathematics, ETH Zürich. Birkhäuser, Basel (1993)

17. Ritt, R.K.: A condition that $\lim _{n \rightarrow \infty} n^{-1} T^{n} n=0$. Proc. Am. Math. Soc. 4, 898-899 (1953)

18. Seyoum, W., Mengestie, T.: Spectral and uniform mean ergodicity of weighted composition operators on Fock-type spaces (2020) (preprint)

19. Shields, A.L.: On Möbius bounded operators. Acta Sci. Math. (Szeged) 40, 371-374 (1978)

20. Ueki, S.: Weighted composition operators on the Fock space. Proc. Am. Math. Soc. 135, 1405-1410 (2007)

Publisher's Note Springer Nature remains neutral with regard to jurisdictional claims in published maps and institutional affiliations. 